\title{
Mineralização do nitrogênio em solos tratados com lodos de curtume
}

\author{
Marco Aurélio Kondracki de Alcântara ${ }^{(1)}$, Vicente de Aquino Neto ${ }^{(2)}$, Otávio Antonio de Camargo ${ }^{(3)}$ \\ e Heitor Cantarella ${ }^{(3)}$
}

\begin{abstract}
(1)Universidade de São Paulo, Escola de Engenharia de Lorena, Dep. de Biotecnologia, Rodovia Itajubá-Lorena, Km 74,5, Caixa Postal 116, CEP 12600-970 Lorena, SP. E-mail: maka@debiq.eel.usp.br ${ }^{(2)}$ Companhia de Tecnologia de Saneamento Ambiental, Setor de Apoio Técnico em Áreas Contaminadas, Av. Prof. Frederico Hermann Júnior, 345, CEP 05489-900 São Paulo, SP. E-mail: vicentea@cetesb.sp.gov.br (3)Instituto Agronômico, Centro de Pesquisa e Desenvolvimento de Solos e Recursos Agroambientais, Caixa Postal 28, CEP 13001-970 Campinas, SP. E-mail: ocamargo@iac.sp.gov.br, cantarella@iac.sp.gov.br
\end{abstract}

Resumo - O objetivo deste trabalho foi avaliar, em laboratório, a mineralização líquida do $\mathrm{N}$ orgânico de dois lodos de curtume em solos. Um Latossolo Vermelho acriférrico e um Latossolo Vermelho-Amarelo distrófico receberam quatro doses de cada lodo $\left(0,595,1.190\right.$ e $\left.1.785 \mathrm{~kg} \mathrm{ha}^{-1} \mathrm{de} \mathrm{N}\right)$ : do efluente de caleiro (LCL), com concentração baixíssima de crômio e do decantador primário (LCR), com $17 \mathrm{~g} \mathrm{~kg}^{-1} \mathrm{de}$ Cr. As misturas solos+lodos foram lixiviadas após incubação por períodos crescentes até 132 dias em colunas de percolação. Um modelo cinético de primeira ordem descreveu a mineralização líquida do $\mathrm{N}\left(\mathrm{R}^{2}=0,967 * *\right.$ a $\left.0,998^{* *}\right)$. Aplicações de LCR, em comparação às de LCL, diminuíram a porcentagem acumulada do $\mathrm{N}$ mineralizado proveniente do lodo (médias de 4 e $35,5 \%$ ), reduziram o valor da taxa constante de mineralização (médias de 0,0048 e $0,028 \mathrm{dia}^{-1}$ ) e aumentaram substancialmente o tempo de meia-vida $\left(\mathrm{T}_{1 / 2}\right)$ (variação de 100 a 267 e de 19 a 29 dias), o que indica inibição da mineralização do $\mathrm{N}$, possivelmente em decorrência das elevadas concentrações de $\mathrm{Cr}$ daquele lodo. Uma análise detalhada das curvas de mineralização com a aplicação de LCR apontou a existência de três fases, que provavelmente representam adaptações sucessivas da microbiota a diferentes frações recalcitrantes do lodo.

Termos para indexação: curtume, mineralização, Latossolo.

\section{Nitrogen mineralization in soils treated with tannery sludge}

\begin{abstract}
The objective of this work was to evaluate nitrogen mineralization in two tannery sludges in soils. A Rhodic Acrustox and a Typic Haplustox received four levels of each sludge $\left(0,595,1,190\right.$ and 1,785 $\left.\mathrm{kg} \mathrm{ha}^{-1} \mathrm{~N}\right)$ : a liming sludge (LCL), comprising very low chromium contents and a primary sludge (LCR), with $17 \mathrm{~g} \mathrm{~kg}^{-1} \mathrm{Cr}$. Soil-sludge mixture was placed in percolation tubes and periodically leached after incubation up to 132 days. First order kinetic model described the nitrogen net mineralization $\left(\mathrm{R}^{2}=0.967^{* *}\right.$ to $\left.0.998^{* *}\right)$. Compared to LCL, the addition of LCR caused a decrease in the percentages of the accumulated mineralized nitrogen from sludges (averages 35.5 and $4.0 \%$, respectively), a decrease in the mineralization rates constants (averages 0.028 and 0.0048 day $^{-1}$ ) and substantially increased the $\mathrm{T}_{1 / 2}$ (ranging from 267 to 100 and from 29 to 19 days), indicating $\mathrm{N}$ mineralization inhibition, possible due to the high chromium content of that sludge. A detailed analysis of mineralization curves with LCR applications pointed that there are three phases that probably represent successive adaptations of the microbiota to different recalcitrant fractions.
\end{abstract}

Index terms: chromium, mineralization, Oxisol.

\section{Introdução}

$\mathrm{O}$ teor de $\mathrm{N}$ em lodos gerados pela indústria do couro é frequientemente o fator que determina a quantidade de lodo que poderá ser aplicada ao solo (A General..., 2001). O N orgânico contido nesses lodos não é prontamente disponibilizado para as plantas. Três a $72 \%$ do $\mathrm{N}$ orgânico contido em lodos estão sujeitos à mineralização, que, por sua vez, depende do tipo de lodo, dose aplicada, características do solo e procedimento experimental (Alves et al., 1999; Silva et al., 1999; Munn et al., 2000; Gonçalves et al., 2001; Boeira et al., 2002; Rangel et al., 2006).

Tal resíduo pode apresentar elevadas concentrações de $\mathrm{Cr}$ trivalente, $\mathrm{Cr}$ (III). $\mathrm{O}$ acúmulo de metais pesados no solo pode interferir na atividade microbiana e conseqüentemente na mineralização do N. Os resultados 
encontrados são diversos, e até contraditórios. BarajasAceves \& Dendooven (2001) aplicaram um lodo de curtume contendo $\mathrm{Cr}$ em solos de regiões semi-áridas do México e observaram aumento na mineralização de $\mathrm{C}$ e N. Embora não tenham sido observados efeitos inibitórios no funcionamento biológico do solo, os autores advertem que estudos posteriores para verificar efeitos ambientais de longo prazo seriam necessários. AlvarezBernal et al. (2006) irrigaram, durante 25 anos, solos agrícolas com águas residuárias de curtumes contendo diversos metais (inclusive $\mathrm{Cr}$ ). Embora aparentemente não tenham ocorrido impactos desfavoráveis em diversas características de solo e na biomassa microbiana, houve inibição da oxidação de $\mathrm{NO}_{2}^{-}$, o que indica que o funcionamento biológico do solo poderia ter sido afetado. Munn et al. (2000), ao aplicarem lodo de esgoto contendo diversos metais, entre eles $\mathrm{Cr}, \mathrm{Pb}$ e $\mathrm{Cd}$ nas concentrações de 187,80 e $2,5 \mathrm{mg} \mathrm{kg}^{-1}$, respectivamente, detectaram aumento na mineralização e na disponibilidade de $\mathrm{N}$ no solo com o aumento da dose de lodo aplicado.

$\mathrm{O}$ teor de manganês do solo também influencia a química do Cr. Em solos com altos teores de formas oxidadas de manganês (Mn(IV) e Mn(III)), também denominadas de facilmente redutíveis, estas tenderão a se reduzir, criando um ambiente favorável à oxidação do $\mathrm{Cr}(\mathrm{III})$ a $\mathrm{Cr}(\mathrm{VI})$. Além disso, a oxidação do Cr pelo manganês ocorre preferencialmente à oxidação desse metal pela matéria orgânica do solo (Canali et al., 1997). Bartlett \& James (1980), em amostras de solo recentemente umedecidas, observaram que a quantidade de Cr oxidada foi proporcional aos teores de manganês reduzido no solo. Utilizando um solo Gleissolo Melânico com elevados teores de manganês, com potencial para oxidar Cr(III), James \& Bartlett (1984) observaram inibição na formação de nitrato ao adicionar $52 \mathrm{mg} \mathrm{kg}^{-1} \mathrm{de} \mathrm{Cr}$ (III) ou $\mathrm{Cr}(\mathrm{VI})$, ao solo. Segundo os autores, a presença de $\mathrm{Cr}(\mathrm{VI})$ em solução, adicionado ou formado no solo pela oxidação da forma trivalente, foi responsável pela inibição da nitrificação, tendo o $\mathrm{Cr}$ (III) pouca influência nesta transformação. A redução dessas formas de manganês facilmente reduzíveis depende, entre outros fatores, da umidade do solo. Assim, a capacidade de oxidar Cr(III) altera-se quando o solo é secado antes da sua utilização.

As informações disponíveis no Brasil sobre a mineralização do $\mathrm{N}$ presente em lodos de curtume ainda não são suficientemente conclusivas para oferecer subsídios ao uso desses resíduos em solos agrícolas.
O objetivo deste trabalho foi avaliar a mineralização líquida do N orgânico de lodos de curtume em solos.

\section{Material e Métodos}

As amostras de solo foram caracterizadas química e fisicamente, conforme métodos descritos por Camargo et al. (1986) e Raij et al. (2001). Os teores totais de $\mathrm{Mn}$ e de $\mathrm{Cr}$ foram determinados pelo método SW-846-3050 (USEPA, 2004). Foram utilizadas amostras dos horizontes A de dois solos do Estado de São Paulo: Latossolo Vermelho acriférrico (LV), textura argilosa, e Latossolo Vermelho-Amarelo distrófico (LVA), textura média (Embrapa, 1999). O LVA e o LV apresentaram, respectivamente, as seguintes características: C orgânico total, 11,6 e 14,0 $\mathrm{g} \mathrm{kg}^{-1} ; \mathrm{Mn}$ facilmente redutível: $0,00 \mathrm{e} 191,7 \mathrm{mg} \mathrm{kg}^{-1}$; Mn total: 41,00 e 717,0 $\mathrm{mg} \mathrm{kg}^{-1}$; Crtotal: $<0,30$ e 34,0 $\mathrm{mg} \mathrm{kg}^{-1}$; CTC efetiva: 5,2 e $8,8 \mathrm{mmol}_{\mathrm{c}} \mathrm{dm}^{-3}$; P resina: $3,0 \mathrm{e} 24,0 \mathrm{mg} \mathrm{dm}^{-3}$; argila: 120 e $560 \mathrm{~g} \mathrm{~kg}^{-1}$ e pH em $\mathrm{H}_{2} \mathrm{O}$ (1:1): 4,0 e 5,0. Um dos fatores importantes na escolha desses solos refere-se aos teores de Mn facilmente redutível.

Os solos foram coletados úmidos, passados em peneira com malha de $2 \mathrm{~mm}$ e acondicionados em recipientes de PVC hermeticamente fechados para manter-se a umidade natural. Desta forma, procurouse manter o estado de oxidação dos óxidos de $\mathrm{Mn}$ presentes.

Foram coletadas amostras de dois tipos de lodo produzidos por um curtume localizado em Leme, SP, resultantes de etapas diferentes do processo de curtição do couro, denominados de LCR e LCL. O LCR foi produzido no decantador primário, resultante da reunião dos efluentes gerados no processo, até mesmo o da etapa de curtimento ao Cr. O LCL foi produzido pela precipitação do efluente gerado na etapa de caleiro. Nessa etapa, a precipitação foi realizada por meio do abaixamento do $\mathrm{pH}$ do efluente até 5,5. Após a precipitação e separação do lodo, $\mathrm{o} \mathrm{pH}$ da mistura foi elevado até 8,0 (pH normal do efluente). Os dois lodos (LCR e LCL) foram utilizados na forma bruta, isto é, sem terem sido submetidos a processo de estabilização. A escolha desses dois lodos foi feita por causa da possibilidade de o processo de mineralização do $\mathrm{N}$ contido no LCR ser inibido em relação ao LCL, em decorrência dos maiores teores de $\mathrm{Cr}$ daquele lodo. As características químicas dos lodos utilizados são apresentadas na Tabela 1. 
Os lodos coletados foram passados em peneira com malha de $10 \mathrm{~mm}$, homogeneizados, armazenados em recipientes de polietileno de 50 L e mantidos em câmara fria a $4^{\circ} \mathrm{C}$ até o momento de sua utilização, quando foram secados em estufa a $40^{\circ} \mathrm{C}$, até a redução da umidade para um valor entre 70 e $80 \%$ aproximadamente. Eles foram caracterizados determinando-se: $\mathrm{pH}$ em água (1:1), $\mathrm{N}$ total (digestão com $\mathrm{H}_{2} \mathrm{SO}_{4}$ e $\mathrm{K}_{2} \mathrm{SO}_{4}$, com modificação envolvendo ácido salicílico para incluir $\mathrm{NO}_{3}{ }^{-}$) (Eaton et al., 2005), umidade ( $\left.105^{\circ} \mathrm{C}\right), \mathrm{K}, \mathrm{Na}, \mathrm{Ca}, \mathrm{Mg}, \mathrm{Mn}$ e $\mathrm{Cr}$ totais pelo método da SW-846-3050B (USEPA, 2004), C orgânico total pelo método Walkley-Black (Camargo et al., 1986) e P total (Eaton et al., 2005).

Em ambos os solos, foram aplicadas quatro doses de cada lodo úmido (TESTEM, LCR-1; LCR-2; LCR-3 para o LCR e TESTEM, LCL-1; LCL-2; LCL-3 para o LCL), de modo a fornecer 595, 1.190 e $1.785 \mathrm{~kg} \mathrm{ha}^{-1} \mathrm{de}$ $\mathrm{N}$, considerando-se a profundidade de $20 \mathrm{~cm}$. O tratamento TESTEM correspondeu à incubação dos solos sem adição de lodo. Essas quatro doses corresponderam a uma adição de $0,256,512$ e $768 \mathrm{mg} \mathrm{kg}^{-1} \mathrm{de} \mathrm{N}$ ao LV (densidade $1,162 \mathrm{~kg} \mathrm{dm}^{-3}$ ) e de 0, 226, 452 e $678 \mathrm{mg} \mathrm{kg}^{-1}$ de $\mathrm{N}$ ao LVA (densidade $1,314 \mathrm{~kg} \mathrm{dm}^{-3}$ ). Essas doses de $\mathrm{N}$ são muito superiores às normalmente utilizadas no campo. Dois motivos foram importantes para esse procedimento: o fato de que nem todo o $\mathrm{N}$ presente nos lodos será aproveitado pelas plantas, mesmo num período prolongado; o propósito de adicionar maiores quantidades de $\mathrm{Cr}$ ao solo.

$\mathrm{O}$ pH de cada mistura de solo+lodo foi elevado a 6,5, utilizando-se mistura de $\mathrm{CaCO}_{3}+\mathrm{MgO}$, proporção 3:1,

Tabela 1. Características químicas das amostras dos lodos com base na matéria seca (LCR - lodo do decantador primário; LCL - lodo do caleiro).

\begin{tabular}{lrr}
\hline Características & LCR & LCL \\
\hline Umidade $\left(\mathrm{g} \mathrm{kg}^{-1}\right)$ & 817 & 689 \\
Carbono orgânico total $\left(\mathrm{g} \mathrm{kg}^{-1}\right)$ & 239 & 240 \\
Crômio total $\left(\mathrm{g} \mathrm{kg}^{-1}\right)$ & 17,3 & 0,06 \\
Nitrogênio total $\left(\mathrm{g} \mathrm{kg}^{-1}\right)$ & 31,2 & 59,4 \\
Fósforo total $\left(\mathrm{g} \mathrm{kg}^{-1}\right)$ & 1,06 & 0,89 \\
Potássio $\left(\mathrm{g} \mathrm{kg}^{-1}\right)$ & 1,66 & 1,92 \\
Sódio $\left(\mathrm{g} \mathrm{kg}^{-1}\right)$ & 52,5 & 66,0 \\
Cálcio $\left(\mathrm{g} \mathrm{kg}^{-1}\right)$ & 87,2 & 67,0 \\
Magnésio $\left(\mathrm{g} \mathrm{kg}^{-1}\right)$ & 7,54 & 0,47 \\
Manganês total $\left(\mathrm{g} \mathrm{kg}^{-1}\right)$ & 6,35 & 0,97 \\
pH (adimensional) & 8,0 & 8,0 \\
\hline
\end{tabular}

cujas doses foram estimadas por meio de curvas de neutralização previamente obtidas pela incubação com o corretivo.

O experimento para estimar a mineralização do $\mathrm{N}$ no solo utilizou método adaptado de Stanford \& Smith (1972). Esse método consiste na incubação do solo com diferentes doses de lodo em colunas de percolação, com remoção periódica do $\mathrm{N}$ mineralizado do sistema por meio de lixiviação com uma solução salina diluída. Este procedimento simula a lixiviação e a absorção pelas plantas e evita o acúmulo de substâncias que, direta ou indiretamente, possam inibir o processo de mineralização.

Foram utilizadas 32 colunas de percolação (2 solos X 2 lodos $\mathrm{x} 4$ doses de $\mathrm{N}$ x 2 repetições). Em cada coluna de percolação foram adicionados $40 \mathrm{~g}$ de solo+lodo misturados com $20 \mathrm{~g}$ de esferas de vidro com diâmetro médio de $2 \mathrm{~mm}$, para aumentar a aeração e a permeabilidade. Discos de manta geotêxtil foram colocados na base da coluna e logo acima da superfície do solo a fim de evitar a sua dispersão e compactação pela aplicação da solução a ser lixiviada. As colunas foram tampadas com folha de papel-alumínio com pequeno orifício e envoltos com cartolina preta, para permitir trocas gasosas, reduzir a perda de umidade e impedir o desenvolvimento de vegetais e de fungos entre a amostra de solo e a parede dos tubos. O ensaio foi conduzido em laboratório à temperatura ambiente (25 a $28^{\circ} \mathrm{C}$ ).

As colunas de percolação foram preenchidas com os solos tratados com diferentes doses dos lodos. Depois da montagem, cada coluna recebeu aplicação de $200 \mathrm{~mL}$ de solução $0,01 \mathrm{~mol} \mathrm{~L}^{-1} \mathrm{de} \mathrm{KCl}$, em alíquotas de $20 \mathrm{~mL}$, para remover o $\mathrm{N}$ inorgânico preexistente. Essa adição foi repetida após 7, 14, 34, 48, 62, 77, 90, 104, 118 e 132 dias de incubação. Para manter as condições de aerobiose durante todo o período de incubação, imediatamente após o término de cada lixiviação, foi aplicado uma sucção de $0,067 \mathrm{MPa}$ às colunas. O líquido percolado obtido após cada lixiviação foi analisado para N por destilação (Eaton et al., 2005). As formas de $\mathrm{N}$ nítrico e amoniacal quantificadas no líquido percolado foram somadas e consideradas como $\mathrm{N}$ mineralizado (Nm).

Embora na metodologia originalmente proposta por Stanford \& Smith (1972) tenham sido utilizados $100 \mathrm{~mL}$ de uma solução salina de $\mathrm{CaCl}_{2} 0,01 \mathrm{~mol} \mathrm{~L}^{-1}$, aplicados em alíquotas de 5 a $10 \mathrm{~mL}$, em ensaios prévios observou-se 
que a lixiviação nas condições empregadas foi igualmente capaz de remover todo o amônio existente nos solos acondicionados nas colunas com os diferentes tratamentos. Além disso, após cada lixiviação, os autores aplicaram às colunas $25 \mathrm{~mL}$ de uma solução nutritiva (sem N), de $\mathrm{CaSO}_{4} \cdot 2 \mathrm{H}_{2} \mathrm{O}, \mathrm{MgSO}_{4}, \mathrm{Ca}\left(\mathrm{H}_{2} \mathrm{PO}_{4}\right) \cdot \mathrm{H}_{2} \mathrm{O}$ e $\mathrm{K}_{2} \mathrm{SO}_{4}$, de modo a repor a remoção desses nutrientes. Neste estudo, não houve tal preocupação, o que pode ter influenciado o processo de mineralização do N, uma vez que vários íons foram removidos do sistema. Assim, os resultados obtidos nos diversos tratamentos devem ser analisados comparativamente entre si. Por sua vez, é possível que a não reposição desses íons tenha possibilitado maior aproximação das condições de lixiviação a campo, considerando-se ausência de fertilização de qualquer natureza.

Em virtude de as colunas terem sido lixiviadas previamente, no início do período de incubação (t, 0 dia), não foi recolhido o $\mathrm{N}$ mineralizado no percolado da coluna (Nm, 0). A quantidade de Nm acumulada no solo durante os 132 dias de incubação foi denominada de Nm acumul. Foi também calculada a $\mathrm{Nm} \%$, como sendo a porcentagem que o $\mathrm{Nm}$ acumul representa em relação ao $\mathrm{N}$ aplicado via lodo de curtume, menos o $\mathrm{Nm}$ acumulado na testemunha, de acordo com:

$\mathrm{Nm} \%=100(\mathrm{Nm}$ acumul LODO+SOLO $-\mathrm{Nm}$ acumul TESTEM)/N aplicado lodo

em que $\mathrm{Nm}$ acumul TESTEM corresponde ao $\mathrm{N}$ mineralizado acumulado, determinado no final do experimento no solo, sem aplicação de lodos; $\mathrm{Nm}$ acumul LODO+SOLO corresponde ao $\mathrm{N}$ mineralizado acumulado durante o período total de incubação, no solo que recebeu aplicação de lodo; $\mathrm{N}$ aplicado lodo corresponde ao $\mathrm{N}$ aplicado ao solo via lodo. $\mathrm{O}$ efeito das interações entre tipo de solo e tipo de lodo não foi significativo a $5 \%$ de probabilidade.

Existem vários modelos matemáticos para simular a mineralização do N no solo. Stanford \& Smith (1972) propuseram um modelo para estimar a taxa de mineralização líquida de $\mathrm{N}$, ou seja, a variação na mineralização de acordo com o tempo. Nesse modelo, considera-se que a mineralização do $\mathrm{N}(\mathrm{Nm})$ segue uma reação cinética de primeira ordem:

$\mathrm{N}_{\mathrm{m}}=\mathrm{N}_{0}\left(1-\mathrm{e}^{-\mathrm{kt}}\right)$

em que $\mathrm{Nm}$ é o $\mathrm{N}$ mineralizado; $\mathrm{N}_{0}$ é o $\mathrm{N}$ potencialmente mineralizável; $\mathrm{k}$ é a taxa de mineralização, considerada constante nesse modelo; e t é o tempo de incubação. $\mathrm{O} \mathrm{N}$ mineralizado $(\mathrm{Nm})$, neste caso, corresponde à mineralização líquida, ou seja, ao balanço entre os processos brutos de mineralização e imobilização do N.

A fim de descrever a produção acumulada de $\mathrm{N}$ inorgânico no período experimental, foram ajustadas curvas de mineralização de acordo com o modelo apresentado na expressão (2) aos dados de Nm obtidos experimentalmente em cada tempo de incubação. Esse ajuste foi feito por regressão não linear, utilizando-se o método de Marquadt (quadrados mínimos).

Finalmente, a aplicação do modelo foi detalhada, ainda que de modo incipiente, em um dos tratamentos conduzidos. Esse detalhamento objetivou mostrar comportamentos diferenciados na mineralização do $\mathrm{N}$ ao longo do período experimental. Neste artigo, são apresentados somente os dados obtidos para o solo LV, com aplicação da dose mais baixa de lodo do decantador primário (LCR-1). As quantidades de Nm acumul foram ajustadas em dois níveis de detalhamento. No primeiro nível de detalhamento, mais geral, considerou-se o período experimental total (132 dias). No segundo nível, mais detalhado, as quantidades de $\mathrm{Nm}$ acumul foram ajustadas considerando-se três subperíodos: de 0 a 34, de 34 a 77 e de 77 a 132 dias de incubação. Esses subperíodos foram denominados, respectivamente, de A, B e C.

\section{Resultados e Discussão}

$\mathrm{Na}$ maioria dos tratamentos analisados de ambos os solos, a adição de doses crescentes dos lodos resultou em maiores quantidades de $\mathrm{N}$ mineralizado acumulado do sistema solo-lodo, o que pode ser verificado pelos valores crescentes de $\mathrm{Nm}$ acumul (Tabela 2). Porém, quando a mineralização é comparada em termos de porcentagem em relação ao fornecido pelo lodo $(\mathrm{Nm} \%)$, tal aumento não pôde ser evidenciado.

Quando se leva em conta os dados de Nm\%, percebese que o comportamento foi diferenciado em decorrência do tipo de lodo aplicado. Quando foi aplicado LCL (lodo de caleiro, com baixa concentração de $\mathrm{Cr}$ ), os valores de Nm\% variaram de 34 a 38\% (média de 35,5\%), considerando ambos os solos. Com a aplicação de LCR (lodo do decantador primário, rico em $\mathrm{Cr}$ ), o $\mathrm{Nm} \%$ variou de -5 a 9\% (média de 4,0\%), considerando ambos os solos. Ou seja, com a aplicação de LCR a mineralização do $\mathrm{N}$ foi significativamente menor do que com a aplicação de LCL. Uma explicação para tais resultados é a de que o $\mathrm{Cr}$ existente em concentrações elevadas 
no LCR estaria inibindo a mineralização do N. Contudo, o grau de inibição verificado e o estado de oxidação do Cr responsável por este fato não é consenso entre trabalhos conduzidos por outros pesquisadores (BarajasAceves \& Dendooven, 2001). Em algumas situações, o decréscimo na mineralização pode ter sido causado por uma ação direta do metal sobre a população microbiana, ao ser aplicado na forma de sal inorgânico. Canali et al. (1997) citam trabalhos nos quais a atividade de células microbianas pode ser inibida quando em contato com $\mathrm{Cr}$ na forma de sal inorgânico, e este processo é normalmente associado a efeitos tóxicos. Também concluem que, embora a atividade seja inibida, a população de bactérias no solo muda muito pouco na presença de elevadas concentrações do metal.

Além da inibição da atividade microbiana, é possível que, nos solos com aplicação de LCR, tenha ocorrido formação de complexos de difícil degradação, entre o Cr e o material orgânico, predominantemente formado de proteínas animais. Canali et al. (1997) comentam que uma das possíveis causas para a diminuição na produção de $\mathrm{CO}_{2}$ por microrganismos em solos que recebem adição de $\mathrm{Cr}$ trivalente e hexavalente seria o efeito curtente deste elemento, que une os compostos orgânicos, formando complexos de baixa disponibilidade como fonte de energia e de decomposição muito lenta no solo. Wiseman \& Zibilske (1988) também observaram diferenças entre a taxa de mineralização do $\mathrm{N}$ em solos que receberam um lodo de esgoto de origem doméstica $(24,5 \%)$ e outro com grande contribuição de curtumes $(20,0 \%)$. Os autores atribuíram essa inibição na mineralização à formação de compostos recalcitrantes existentes nos lodos.

Neste experimento, $\mathrm{Nm}$ corresponde à mineralização líquida, ou seja, ao balanço entre as quantidades totais de $\mathrm{N}$ mineralizado (mineralização bruta) e de $\mathrm{N}$ imobilizado (imobilização bruta). Assim, não é possível afirmar se os efeitos na mineralização líquida, discutidos na forma de $\mathrm{Nm} \%$, são decorrentes da inibição na mineralização, ou do aumento intenso na imobilização do $\mathrm{N}$, processo não avaliado neste estudo, porém, menos provável de ter ocorrido.

O modelo exponencial utilizado mostrou bom ajuste aos dados de $\mathrm{Nm}$ obtidos experimentalmente, com $\mathrm{R}^{2}$ entre 0,967 e 0,998 , significativos a $1 \%$ de probabilidade pelo teste de Tukey (Tabela 3 ).

Os valores de $\mathrm{k}$ variaram de 0,0237 a $0,0360 \mathrm{dia}^{-1}$ com média de $0,0282 \mathrm{dia}^{-1}$ para o LCL. Boeira et al. (2002), estudando a mineralização do $\mathrm{N}$ em um Latossolo Vermelho distroférrico que recebeu lodos de esgoto, encontraram variação maior para os valores de $\mathrm{k}$ em relação aos obtidos $\left(0,0069\right.$ a $\left.0,0265 \mathrm{dia}^{-1}\right)$, com média menor, de 0,01385 $\mathrm{dia}^{-1}$. Valores obtidos por Camargo et al. (2004), ao estudarem solos do Rio Grande do Sul, foram maiores em média $\left(0,042 \mathrm{dia}^{-1}\right)$, e apresentaram variação menor $\left(0,030\right.$ a $\left.0,047 \mathrm{dia}^{-1}\right)$. Apenas quanto ao LVA, verificou-se aumento do $\mathrm{k}$, embora de pequena magnitude, com o aumento da dose de lodo aplicada $\left(0,0256\right.$ a $\left.0,0360 \mathrm{dia}^{-1}\right)$, fato que não foi observado para o LV. Boeira et al. (2002), além de não encontrarem aumento do k com o aumento da dose de lodo aplicada, detectaram pequena redução nos valores desse parâmetro de ajuste.

Tabela 2. Nitrogênio aplicado $\left(\mathrm{mg} \mathrm{kg}^{-1}\right), \mathrm{N}$ mineralizado acumulado $\left(\mathrm{mg} \mathrm{kg}^{-1}\right)$ no período de incubação (Nm acumul) e porcentagem do Nm acumul em relação ao N aplicado via lodo (Nm\%) nas colunas do Latossolo Vermelho-Amarelo (LVA) e Latossolo Vermelho (LV). Médias de duas repetições \pm desvio-padrão.

\begin{tabular}{|c|c|c|c|c|c|c|}
\hline \multirow[t]{2}{*}{ Tratamento $^{(1)}$} & \multicolumn{3}{|c|}{ LVA } & \multicolumn{3}{|c|}{ LV } \\
\hline & $\mathrm{N}$ aplicado $^{(2)}$ & $\mathrm{Nm}$ acumulado $^{(3)}$ & $\mathrm{Nm} \%$ & $\mathrm{~N}$ aplicado & Nm acumulado & $\mathrm{Nm} \%$ \\
\hline TESTEM & 0 & $42 \pm 0,6$ & 0 & 0 & $45 \pm 0,9$ & 0 \\
\hline LCL-1 & 226 & $120 \pm 7,3$ & 35 & 256 & $133 \pm 4,2$ & 34 \\
\hline LCL-2 & 452 & $204 \pm 28,5$ & 36 & 512 & $237 \pm 24,3$ & 38 \\
\hline LCL-3 & 678 & $278 \pm 7,1$ & 35 & 768 & $310 \pm 7,0$ & 35 \\
\hline LCR-1 & 226 & $31 \pm 2,2$ & -5 & 256 & $55 \pm 0,4$ & 4 \\
\hline LCR-2 & 452 & $67 \pm 8,3$ & 6 & 512 & $71 \pm 13,1$ & 5 \\
\hline LCR-3 & 678 & $77 \pm 9,0$ & 5 & 768 & $113 \pm 3,2$ & 9 \\
\hline
\end{tabular}

(1)TESTEM: testemunha, solo sem aplicação de lodos; LCL: lodo de caleiro; LCR: lodo do decantador primário. (2)N aplicado: N aplicado ao solo via lodos. ${ }^{(3)} \mathrm{Nm}$ acumul: $\mathrm{N}$ mineralizado nos solos, acumulado no período de incubação considerado. 
Quanto ao LCR, os valores de $\mathrm{k}$ foram substancialmente menores em relação ao LCL, sendo em média da ordem de 0,0048 dia $^{-1}$. Observa-se também variação acentuada nos valores deste parâmetro no LVA $\left(0,0026\right.$ a $\left.0,0069 \mathrm{dia}^{-1}\right)$ e no LV $\left(0,0046\right.$ a $\left.0,0052 \mathrm{dia}^{-1}\right)$. Evidentemente, como o modelo aplicado é exponencial, esta variação no $\mathrm{k}$ não foi linearmente proporcional ao aumento da dose de lodo aplicada. Tais diferenças entre as taxas de mineralização dos lodos estudados podem estar relacionadas à presença de teores elevados de $\mathrm{Cr}$ no LCR, conforme discutido para os dados apresentados na Tabela 2. Na aplicação agrícola, uma baixa taxa de mineralização dificultaria a utilização desse lodo em culturas agrícolas devido à baixa taxa de mineralização por um período superior a 120 dias.

$\mathrm{O} \mathrm{T}_{1 / 2}$ é uma estimativa do tempo necessário para que metade do $\mathrm{N}$ potencialmente mineralizável $\left(\mathrm{N}_{0}\right)$ presente no lodo seja mineralizado. Quanto ao LCR, o $\mathrm{T}_{1 / 2}$ variou entre 100 a 267 dias, valores superiores aos encontrados com a aplicação de LCL, que em média apresentou $\mathrm{T}_{1 / 2}$ de 25 dias, numa amplitude de variação muito menor (19 a 29 dias). Outra observação interessante foi a diferença verificada entre os valores de $\mathrm{T}_{1 / 2}$ dos tratamentos testemunhas, sem adição de lodo, quando se observou para o LVA valor de 146 dias, muito superior ao verificado para o LV, de 73 dias. Esta diferença, provavelmente, deve-se aos diferentes manejos agrícolas, de cada uma das áreas em que os solos foram coletados. O LVA foi coletado em área de reflorestamento com eucaliptos, portanto, recebendo contribuição de materiais mais dificilmente degradáveis do que os encontrados no LV, coletado em área de pasto natural com gramíneas. Observação semelhante foi realizada por Gama-Rodrigues et al. (1997), ao estudarem a biomassa microbiana de $\mathrm{C}$ e $\mathrm{N}$ em solos reflorestados com diferentes espécies florestais.

Os valores de $\mathrm{N}$ potencialmente mineralizável $\left(\mathrm{N}_{0}\right)$ nos solos tratados com LCL (123 a $317 \mathrm{mg} \mathrm{kg}^{-1}$ ) foram superiores aos observados nos solos tratados com LCR (107 a $220 \mathrm{mg} \mathrm{kg}^{-1}$ ), além de apresentarem meia-vida $\left(\mathrm{T}_{1 / 2}\right.$ ) muito menor (19 a 29 dias para os primeiros e 100 a 267 dias para os últimos). Esses dados indicam que o solo tratado com LCL potencialmente fornecerá mais $\mathrm{N}$ e em um intervalo de tempo mais curto do que os tratados com LCR, rico em $\mathrm{Cr}$.

Mesmo nos solos tratados com o LCL, a quantidade de $\mathrm{N}_{0}$, calculada segundo Stanford \& Smith (1972), representa pequena parte do $\mathrm{N}$ contido no lodo. Subtraindo-se os valores de $\mathrm{N}_{0}$ das amostras de solo+lodo, daqueles da testemunha e expressando-se $\mathrm{N}_{0}$ em termos de porcentagem do $\mathrm{N}$ presente no lodo, observa-se que o $\mathrm{N}$ potencialmente mineralizável do LCL equivale a 13 a $28 \%$ do $\mathrm{N}$ do lodo no solo LVA e 29 a $33 \%$ no solo LV. Os valores correspondentes para o LCR foram 7 a $13 \%$ para o solo LVA e 18 a $21 \%$ para o LV. Assim, a maior parte do $\mathrm{N}$ orgânico desses resíduos acrescentados ao solo não seria facilmente liberada em formas inorgânicas, a curto prazo.

As quantidades acumuladas de $\mathrm{N}$ orgânico mineralizado (mineralização líquida acumulada) no LVA e no LV (Figura 1) evidenciam crescimento assintótico da quantidade de $\mathrm{N}$ acumulada ao longo do período de incubação de 132 dias.

Em ambos os solos, os tratamentos com aplicação de LCL resultaram mineralização líquida do $\mathrm{N}$ rápida $\mathrm{e}$ crescente até aos 34 dias. No LVA, em média, 70\% da quantidade total acumulada ao longo do experimento foi

Tabela 3. Coeficientes de determinação $\left(\mathrm{R}^{2}\right)$, parâmetros de ajustes $\mathrm{k}$ e $\mathrm{N}_{0}$ obtidos com o modelo cinético de primeira ordem ${ }^{(1)}$ e tempos de meia-vida $\left(\mathrm{T}_{1 / 2}\right)$ do N potencialmente mineralizável no Latossolo Vermelho-Amarelo (LVA) e no Latossolo Vermelho (LV).

\begin{tabular}{|c|c|c|c|c|c|c|c|c|}
\hline \multirow[t]{2}{*}{ Tratamento $^{(2)}$} & \multicolumn{4}{|c|}{ LVA } & \multicolumn{4}{|c|}{ LV } \\
\hline & $\mathrm{R}^{2}$ & $\mathrm{k}$ & $\mathrm{N}_{0}$ & $\mathrm{~T}_{1 / 2}$ & $\mathrm{R}^{2}$ & $\mathrm{k}$ & $\mathrm{N}_{0}$ & $\mathrm{~T}_{1 / 2}$ \\
\hline & & $\left(\operatorname{dia}^{-1}\right)$ & $\left(\mathrm{mg} \mathrm{kg}^{-1}\right)$ & (dia) & & $\left(\operatorname{dia}^{-1}\right)$ & $\left(\mathrm{mg} \mathrm{kg}^{-1}\right)$ & (dia) \\
\hline TESTEM & $0,990 * *$ & 0,0048 & 93 & 146 & $0,983 * *$ & 0,0096 & 60 & 73 \\
\hline LCL-1 & $0,996^{* *}$ & 0,0256 & 123 & 27 & $0,996 * *$ & 0,0264 & 133 & 26 \\
\hline LCL-2 & $0,988 * *$ & 0,0315 & 212 & 22 & $0,997 * *$ & 0,0261 & 242 & 27 \\
\hline LCL-3 & $0,998 * *$ & 0,0360 & 282 & 19 & $0,993 * *$ & 0,0237 & 317 & 29 \\
\hline LCR-1 & $0,967 * *$ & 0,0026 & 108 & 267 & $0,969 * *$ & 0,0047 & 107 & 146 \\
\hline LCR-2 & $0,987 * *$ & 0,0069 & 114 & 100 & $0,963 * *$ & 0,0046 & 140 & 149 \\
\hline LCR-3 & $0,991 * *$ & 0,0045 & 180 & 153 & $0,990 * *$ & 0,0052 & 220 & 134 \\
\hline
\end{tabular}

(1) Modelo: $\mathrm{N}_{\mathrm{m}}=\mathrm{N}_{0}\left(1-\mathrm{e}^{-\mathrm{kt}}\right)$, em que $\mathrm{N}_{\mathrm{m}}$ é o $\mathrm{N}$ mineralizado; $\mathrm{N}_{0}$ é o $\mathrm{N}$ potencialmente mineralizável; e $\mathrm{k}$ é a taxa de mineralização. ${ }^{(2)}$ TESTEM: testemunha, solo sem aplicação de lodos; LCL: lodo de caleiro; LCR: lodo do decantador primário. **Significativo a $1 \%$ de probabilidade pelo teste de Tukey. 
mineralizada nesse período. Já no LV, houve mineralização de $61 \%$ do $\mathrm{N}$ nesse mesmo período. A partir deste ponto, a taxa de mineralização foi decaindo gradualmente. Comportamento semelhante para lodos de esgoto com elevado $\mathrm{N}_{0}$ foi observado por Boeira et al. (2002). O mesmo comportamento não foi observado para o LCR, que continha elevada concentração de $\mathrm{Cr}$. Neste caso a mineralização ocorreu de forma mais uniforme e lenta.

No que se refere ao modelo proposto ter descrito adequadamente os dados de mineralização do $\mathrm{N}$ (coeficientes de determinação significativos a 1\%) (Tabela 3), tais resultados devem ser analisados com cautela. Na Figura 2, detalhou-se a curva de mineralização do $\mathrm{N}$ no tratamento LCR-1 aplicado ao LV. Tais ajustes detalhados da mineralização do $\mathrm{N}$ foram feitos também para outros tratamentos, com comportamento semelhante ao da Figura 2.

Podem ser identificadas três possíveis tendências dos dados experimentais (A, B e C), quanto à taxa de mineralização do N (Figura 2). Todas as tendências apresentaram comportamento cinético de primeira ordem, semelhante ao proposto por Stanford \& Smith (1972). Comportamento semelhante foi obtido para os demais tratamentos com aplicação de LCR. As três curvas provavelmente se referem a frações distintas de $\mathrm{N}$ orgânico quanto à resistência à degradação, em razão da intensidade da sua interação com o $\mathrm{Cr}$, formando complexos estáveis com o material de origem orgânica, dificultando a ação dos microrganismos, objetivo básico do uso do curtimento de peles animais. As fases identificadas provavelmente representam adaptações sucessivas da população microbiana a estas diferentes frações recalcitrantes, adaptações que vão se sucedendo ao longo do tempo pela seleção natural dos microrganismos mais eficientes na obtenção da energia disponível.

Portanto, nesta situação, o melhor ajuste aos dados experimentais seria alcançado por meio de um modelo mais complexo em relação ao proposto por Stanford \& Smith (1972) e mesmo ao proposto por Molina et al. (1980), que subdividiram o N orgânico contido em lodos de esgoto, em duas frações, uma facilmente degradável e outra recalcitratante. Nos lodos de curtume gerados na decantação primária, esta fração recalcitrante deveria ser subdividida em outras frações, com diferentes valores de taxa de mineralização.

Esse comportamento da curva de mineralização, em vários estágios sucessivos, se aplicou ao LCR, que apresentou altos valores de $\mathrm{T}_{1 / 2}$ - média de $173 \mathrm{e}$ 143 dias, respectivamente, para os solos LVA e LV. Em tais casos a assíntota só seria atingida com um período de incubação mais longo do que o necessário para os solos tratados com LCL (Figura 1). As curvas de mineralização obtidas com esse último lodo, com valores mais baixos de $\mathrm{T}_{1 / 2}$ (médias de 23 e 27 dias para o LVA e LV, respectivamente), refletem a predominância de uma fração de $\mathrm{N}$ com taxa de mineralização mais rápida e, provavelmente, mais homogênea.
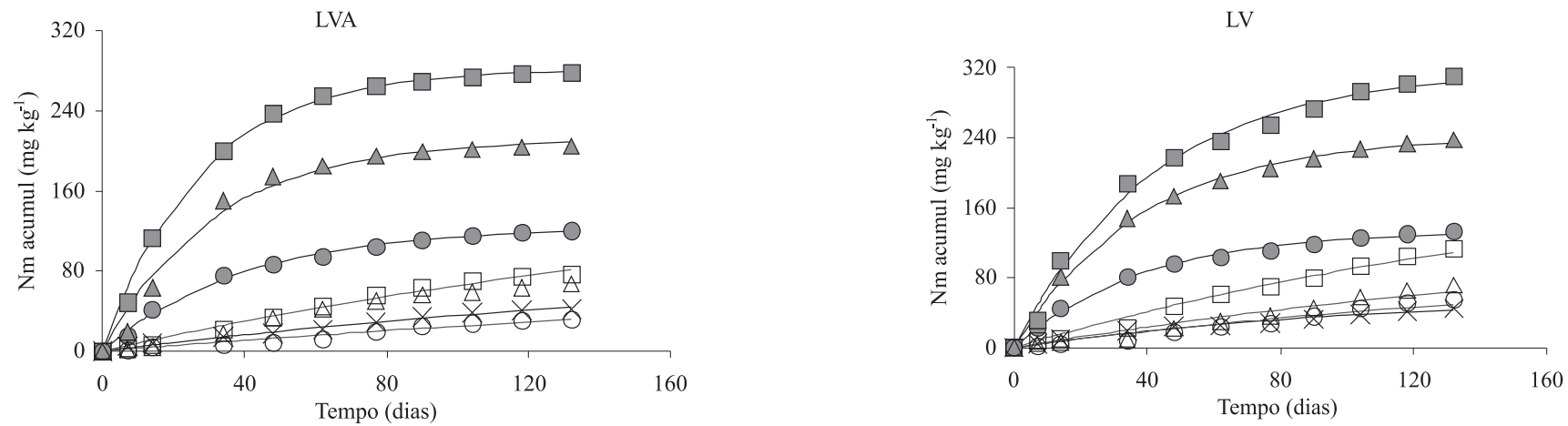

$\times$ TESTEM OLCL-1 $\triangle$ LCL-2 $\square$ LCL-3 OLCR-1 $\triangle$ LCR-2 $\square$ LCR-3

Figura 1. Nitrogênio mineralizado acumulado (Nm acumul) no Latossolo Vermelho-Amarelo (LVA) e Latossolo Vermelho (LV), tratados com diferentes doses de lodo do caleiro (LCL) e de lodo do decantador primário (LCR). TESTEM: testemunha, sem aplicação de lodos. Os pontos correspondem aos dados obtidos experimentalmente. Linhas contínuas: ajustes, considerando-se uma reação cinética de $1 \underline{a}$ ordem, $\mathrm{N}_{\mathrm{m}}=\mathrm{N}_{0}\left(1-\mathrm{e}^{-\mathrm{kt}}\right)$, em que $\mathrm{N}_{0}$ é o $\mathrm{N}$ potencialmente mineralizável e t é o tempo de incubação. 


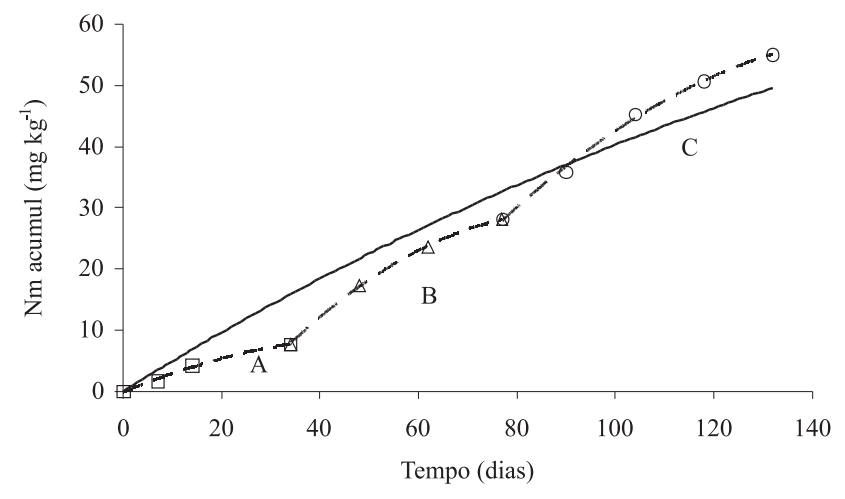

Figura 2. Curvas de mineralização (Nm) do $\mathrm{N}$ orgânico no Latossolo Vermelho (LV), com aplicação da dose 1 do lodo do decantador primário (LCR-1). Linha sólida: calculada por regressão não-linear, assumindo-se uma reação cinética de 1ํㅡ ordem. A, B e C identificam diferentes frações de N orgânico propostas, quanto à dificuldade de mineralização.

Finalmente, os dados de mineralização alcançados são decorrentes de médias entre duas repetições apenas, o que resultou em coeficiente de variação alto $(24,48 \%)$. Contudo, as tendências quanto aos diferentes fatores solos, doses e tipos de lodos - foram claras, o que não deve ter limitado a interpretação dos resultados.

\section{Conclusões}

1. As diferenças na mineralização entre os lodos de curtume, lodo do caleiro (LCL) e do decantador primário (LCR), indicam que a presença de elevados teores de Cr no LCR diminui a velocidade de mineralização do $\mathrm{N}$ orgânico.

2. O modelo de Stanford \& Smith se ajusta adequadamente aos dados experimentais para o lodo do caleiro (LCL), mas não para os do lodo do decantador primário, com alta concentração de $\mathrm{Cr}$ (LCR), o que indica que a mineralização do $\mathrm{N}$ neste caso não segue uma equação cinética de primeira ordem ou que requer um modelo mais complexo, considerando as diferentes frações presentes, com diferentes graus de recalcitrância.

\section{Referências}

A GENERAL guide to environmental permitting in South Carolina. Columbia: South Carolina Dept. of Health and Environmental Control, 2001. 89p.

ALVAREZ-BERNAL，D.; CONTRERAS-RAMOS，S.M.; TRUJILLO-TAPIA, N.; OLALDE-PORTUGAL, V.; FRÍASHERNÁNDEZ, J.T.; DENDOOVEN, L. Effects of tanneries waste- water on chemical and biological soil characteristics. Applied Soil Ecology, v.33, p.269-277, 2006.

ALVES, G.D.; SAMPAIO, E.V.S.B.; SALCEDO, I.H.; SILVA, V.M. Potencial de mineralização de $\mathrm{N}$ e de $\mathrm{C}$ em vinte solos de Pernambuco. Revista Brasileira de Ciência do Solo, v.23, p.245-256, 1999.

BARAJAS-ACEVES, M.; DENDOOVEN, L. Nitrogen, carbon and phosphorus mineralization in soils from semi-arid highlands of Central Mexico amended with tannery sludge. Bioresource Technology, v.77, p.121-130, 2001.

BARTLETT, R.J.; JAMES, B.R. Studying dried, stored soil samples - some pitfalls. Soil Science Society of America Journal, v.44, p.721-724, 1980.

BOEIRA, R.C.; LIGO, M.A.V.; DYNIA, J.F. Mineralização de nitrogênio em solo tropical tratado com lodo de esgoto. Pesquisa Agropecuária Brasileira, v.37, p.1639-1647, 2002.

CAMARGO, F.A.O.; GIANELLO, C.; TEDESCO, M.J. Soil nitrogen availability evaluated by kinetic mineralization parameters. Communications in Soil Science and Plant Analysis, v.35, p.1293-1307, 2004.

CAMARGO, O.A.; MONIZ, A.C.; JORGE, J.A.; VALADARES, J.M.A.S. Métodos de análise química, mineralógica e física de solos do Instituto Agronômico de Campinas. Campinas: Instituto Agronômico, 1986. 94p. (Boletim técnico, 106).

CANALI, S.; TITTARELLI, F.; SEQUI, P. Chromium environmental issues. Milão: Franco Angeli, 1997. 295p.

EATON, A.D.; CLESCERI, L.S.; RICE, E.W.; GREENBERG, A.B. (Ed.). Standard methods for the examination of water and wastewater. $21^{\text {st }}$ ed. Washington: American Public Health Association, American Water Works Association and Water Environment Federation, 2005. 1368p.

EMBRAPA. Serviço Nacional de Levantamento e Conservação de Solos (Rio de Janeiro, RJ). Sistema brasileiro de classificação de solos. Brasília: Embrapa-SPI; Rio de Janeiro: Embrapa-CNPS, 1999. 412p.

GAMA-RODRIGUES, E.F. da; GAMA-RODRIGUES, A.C. da; BARROS, N.F. Biomassa microbiana de carbono e de nitrogênio de solos sob diferentes coberturas florestais. Revista Brasileira de Ciência do Solo, v.21, p.361-365, 1997.

GONÇALVES, J.L.M.; MENDES, K.C.F.S.; SASAKI, C.M. Mineralização de nitrogênio em ecosssistemas florestais naturais e implantados do Estado de São Paulo. Revista Brasileira de Ciência do Solo, v.25, p.601-616, 2001.

JAMES, B.R.; BARTLETT, R.J. Plant-soil interactions of chromium. Journal of Environmental Quality, v.13, p.67-70, 1984.

MOLINA, J.A.E.; CLAPP, C.E.; LARSON, W.E. Potentially mineralizable nitrogen in soil: the simple exponential model does not apply for the first 12 weeks of incubation. Soil Science Society of America Journal, v.44, p.442-443, 1980.

MUNN, K.J.; EVANS, J.; CHALK, P.M. Mineralization of soil and legume nitrogen in soils treated with metal-contaminated sewage sludge. Soil Biology and Biochemistry, v.32, p.2031-2043, 2000.

RAIJ, B. van; ANDRADE, J.C. de; CANTARELLA, H.; QUAGGIO, J.A. (Ed.). Análise química para avaliação da 
fertilidade de solos tropicais. Campinas: Instituto Agronômico, 2001. 285p.

RANGEL, O.J.P.; SILVA, C.A.; BETTIOL, W.; DYNIA, J.F. Efeito de aplicações de lodos de esgoto sobre os teores de metais pesados em folhas e grãos de milho. Revista Brasileira de Ciência do Solo, v.30, p.583-594, 2006.

SILVA, C.A.; VALE, F.R. do; ANDERSON, S.J.; KOBAL, A.R Mineralização de nitrogênio e enxofre em solos brasileiros sob influência da calagem e fósforo. Pesquisa Agropecuária Brasileira, v.34, p.1679-1689, 1999.
STANFORD, G.; SMITH, S.J. Nitrogen mineralization potentials of soils. Soil Science Society of America Journal, v.36, p.465-472, 1972.

USEPA. Metallic analytes: method 3050B acid digestion of sediments, sludges and soils. In: USEPA. SW-846 online: test methods for evaluating solid wastes: physical/chemical methods. Washington, 2004. Disponível em: <http://www.epa.gov/SW-846/ main.htm>. Acesso em: 3 abr. 2007.

WISEMAN, J.T.; ZIBILSKE, L.M. Effects of sludge application sequence on carbon and nitrogen mineralization in soil. Journal of Environmental Quality, v.17, p.334-339, 1988.

Recebido em 16 de outubro de 2006 e aprovado em 9 de março de 2007 